\title{
Sleep Disturbances in Fibromyalgia Syndrome: Relationship to Pain and Depression
}

\author{
Silvia M. Bigatti, PhD¹, Ann Marie Hernandez, MS ${ }^{1}$, Terry A. Cronan, PhD $^{2}$, and Kevin L. \\ Rand, $\mathbf{P h D}^{1}$ \\ ${ }^{1}$ Indiana University-Purdue University Indianapolis, Indianapolis \\ ${ }^{2}$ San Diego State University, San Diego, California
}

\section{Abstract}

Objective-This study is an examination of sleep, pain, depression, and physical functioning at baseline and 1-year followup among patients with fibromyalgia syndrome (FMS). Although it is clear that these symptoms are prevalent among FMS patients and that they are related, the direction of the relationship is unclear. We sought to identify and report sleep problems in this population and to examine their relationship to pain, depression, and physical functioning.

Methods-Patients diagnosed with fibromyalgia were recruited from a Southern California health maintenance organization and evaluated according to American College of Rheumatology criteria in the research laboratory. Six hundred patients completed the baseline assessment and 492 completed the 1-year assessment. Measures included the Center for Epidemiologic Studies Depression Scale, the McGill Pain Questionnaire, the Pittsburgh Sleep Quality Index, and the Fibromyalgia Impact Questionnaire.

Results-The majority of the sample (96\% at baseline and $94.7 \%$ at 1 year) scored within the range of problem sleepers. Path analyses examined the impact of baseline values on 1-year values for each of the 4 variables. No variable of interest predicted sleep, sleep predicted pain $(\beta=0.13)$, pain predicted physical functioning $(\beta=-0.13)$, and physical functioning predicted depression ( $\beta$ $=-0.10)$.

Conclusion-These findings highlight the high prevalence of sleep problems in this population and suggest that they play a critical role in exacerbating FMS symptoms. Furthermore, they support limited existing findings that sleep predicts subsequent pain in this population, but also extend the literature, suggesting that sleep may be related to depression through pain and physical functioning.

\footnotetext{
(C) 2008, American College of Rheumatology
}

Address correspondence to Silvia M. Bigatti, PhD, Department of Psychology, Indiana University-Purdue University Indianapolis, 402 North Blackford Street, LD 124, Indianapolis, IN 46202. sbigatti@iupui.edu.

AUTHOR CONTRIBUTIONS

Dr. Bigatti had full access to all of the data in the study and takes responsibility for the integrity of the data and the accuracy of the data analysis.

Study design. Bigatti, Cronan.

Acquisition of data. Bigatti, Cronan.

Analysis and interpretation of data. Bigatti, Hernandez, Rand.

Manuscript preparation. Bigatti, Hernandez, Cronan.

Statistical analysis. Bigatti, Rand. 


\section{INTRODUCTION}

Fibromyalgia syndrome (FMS) is a chronic musculoskeletal pain disorder. Although pain is the primary chronic symptom, disturbed sleep is also a major symptom of patients with FMS. Patients report difficulty falling asleep, significantly more nighttime awakenings, and unrefreshing sleep (1). The majority of sleep research in patients with FMS focuses on the quality of sleep, because the amount of sleep does not appear to differ between FMS patients and healthy subjects (2). Analysis of electroencephalograms indicates that patients with FMS take longer to fall asleep and have frequent arousals, extended stage 1 sleep, and little slow wave sleep (1), which may indicate a vigilant arousal state during sleep (3). Furthermore, when depressed, patients with FMS report lower levels of activity and increased sleeping during the day, and significantly more sleep interruptions and movement at night (4).

Sleep problems have been related to both depression and pain among patients with FMS in numerous studies (3); however, the direction of these relationships is not yet well established. The Neuromatrix Theory of Pain (5) suggests that pain is impacted by multidimensional factors, including psychological and general health characteristics of individuals. Sleep problems, which fit into the general health characteristics construct of the theory, may impact the neuromatrix through their negative effects on other systems, such as the immune system. Support for this theory comes from research suggesting that sleep may impact the neuromatrix through its effects on intervening systems. For example, lack of sleep leaves individuals more susceptible to infection (6), elevated resting blood pressure and decreased muscle sympathetic nerve activity (7), and increased activity of the autonomic nervous system (8). Specific to FMS, research suggests that good sleep quality may moderate the relationship between affect and pain (9). Essentially, a good night's sleep increases our ability to resist bouts of pain; poor sleep, on the other hand, especially when chronic, may increase our vulnerability to symptoms.

Nicassio and Wallston (10) evaluated the relationship between sleep problems, pain, and depression over 2 years among patients with rheumatoid arthritis. Their longitudinal analyses indicated that sleep problems did not predict subsequent pain, but pain predicted subsequent sleep problems. In addition, sleep problems did not predict subsequent depression, but pain did.

Affleck et al (11) investigated the temporal sequence of pain and sleep problems among a sample of patients with FMS over a 30-day period, using handheld devices to record sleep quality in the morning and pain throughout the day. Their findings suggested that sleep difficulties the night before predicted increased pain during the day, but daily measures of pain did not predict poorer subsequent sleep.

The difference in findings between Affleck et al (11) and Nicassio and Wallston (10) may have occurred because they studied different patient populations. Sleep may very well be an etiologic factor for FMS symptoms, but not for symptoms in other rheumatic conditions. This was investigated by Moldofsky and Scarisbrick (12), who altered the sleep pattern of 6 healthy subjects to imitate those of FMS patients. All subjects subsequently reported symptoms similar to those reported by patients with FMS. Their study suggested that poor sleep may have a significant effect on the development or exacerbation of FMS symptoms and paved the way for future studies of sleep in patients with FMS.

In the present study, we examined sleep, pain, depression, and physical functioning at baseline and 1 year later among patients with FMS. Because they used a similar research design, we built on the work of Nicassio and Wallston (10) by using a path analysis approach; however, because our sample consisted of FMS patients, we hypothesized that our 
findings would match those of Affleck and colleagues (11), in that baseline sleep would predict subsequent pain. We also predicted that both sleep and pain would predict subsequent depression. We had no specific prediction for physical functioning because this variable has not been examined in relation to sleep in the literature.

\section{PARTICIPANTS AND METHODS}

\section{Participants}

The participants consisted of 600 members of a large health maintenance organization (HMO). They were largely women $(95.3 \%)$ and white $(85 \%)$, with a mean \pm SD age of $53.92 \pm 11.45$ years. Most participants were married $(64.2 \%)$ and almost half were employed (49.5\%). Their average family income was between $\$ 30,000$ and $\$ 50,000$, and $81 \%$ of the sample reported at least some college education. Participants reported experiencing FMS symptoms for a mean \pm SD of $13.8 \pm 13.2$ years.

\section{Measures}

Demographic characteristics—Participants reported their age (in years), highest level of education completed, work status (employed/not employed), ethnicity (white/ethnic minority), and family income.

Sleep quality-The Pittsburgh Sleep Quality Index (PSQI) (13) estimates sleep duration, sleep latency, and the frequency and severity of specific sleep-related problems during the past month. The index comprises 19 items grouped into 7 component scores, each weighted equally on a 4-point Likert scale, ranging from 0 (not during the past month) to 3 ( $>3$ times a week). The component scores are summed to yield a global PSQI score. This score ranges from $0-21$, with higher scores indicating worse quality of sleep. Reliability estimates showed internal consistency of 0.83 for the 7 component scores, and test-retest reliability of 0.85 for the PSQI global scores (13).

Pain assessment-To assess pain we used the Pain Rating Index scale of the McGill Pain Questionnaire (14), which assesses the sensory qualities and cognitive affective characteristics of pain perception. It comprises 78 adjectives divided into 4 subscales: sensory, affective, evaluative pain, and miscellaneous pain. Adjectives are presented in groups of 3-6 and the respondent is instructed to mark the word that best describes his or her pain in the past week. Scores ranged from 0 (mild) to 6 (severe). Mean scale values are obtained from each category and then averaged to yield 1 score (14). Test-retest reliability has been demonstrated previously $(r=0.70)(14)$. Construct validity for the 3 pain dimensions has also been demonstrated (15).

Depression-The Center for Epidemiologic Studies Depression Scale (CES-D) (16) contains 20 items rated on a 4-point Likert scale ranging from 0 (rarely or none of the time) to 3 (most or all of the time), and measures symptoms during the past week. The scale is reliable $(r=0.88)$, internally consistent (alpha coefficients range from $0.80-0.90)$, has moderate test-retest reliability ( $r=0.40$ and greater), and high concurrent and construct validity (16).

Physical functioning score-The Fibromyalgia Impact Questionnaire (FIQ) is a brief, self-administered, 10-item questionnaire that measures physical functioning, depression, work status, sleep, anxiety, pain, stiffness, fatigue, and well-being over the past week in patients with FMS (17). The first item of the FIQ consists of 10 subitems that focus primarily on the patient's ability to perform large muscle tasks (i.e., doing yard work, vacuuming a rug), presented in a Likert-scale-type format ranging from 0 (always able to 
do) to 3 (never able to do). The sum of these 10 subitems is divided by the number of valid scores to provide 1 physical functioning score, which for the purposes of these analyses were recoded so that higher scores reflected better functioning. The scale has previously demonstrated test-retest reliability $(0.56-0.95)$. Both content and construct validity were demonstrated in the development of the scale (17).

\section{Procedure}

Participants were part of a larger study measuring the effects of social support and education on health status, health care utilization, and quality of life in patients with FMS (18). Recruitment was carried out through newspaper advertisements, mass mailings to HMO members, flyers in physicians' offices, and physician referrals. When asked how a participant heard about the study, most reported multiple modalities, making comparisons between patients recruited by the different methods impossible. To be eligible, participants had to be diagnosed by a physician and had to meet American College of Rheumatology criteria for fibromyalgia (19). A trained examiner performed the manual tender point examination after informed consent was obtained. Only participants who met all criteria were retained for this study.

Informed written consent was obtained from all volunteers. The assessments lasted $\sim 1$ hour. Participants completed a battery of questionnaires at the baseline assessment and again 1 year later. After the baseline assessment, subjects were randomly assigned to 1 of 2 interventions (social support or social support and education) or to a no treatment control group. The contents of the educational sessions and the social support tasks were standardized and adapted specifically for FMS. There were no differential changes in health status or psychosocial measures used in the present study by groups, although the social support and education group was less helpless at the 1-year assessment than the other groups (18). Therefore, we collapsed the groups for the present study.

\section{RESULTS}

\section{Completers versus noncompleters}

Of the 600 participants who joined the study, $492(82 \%)$ completed the 1-year assessment. We examined differences between these and the 108 participants who dropped out of the study. There were no significant differences between the participants who continued and those who dropped out in any of the demographic variables $(P>0.05)$, or in sleep, pain, depression, or physical functioning $(P>0.05)$.

\section{Descriptive analyses}

Sleep-Participants scored high on the PSQI at both time points, but significantly higher at baseline than at 1 year (baseline mean \pm SD $11.22 \pm 3.96$, 1-year mean \pm SD $10.75 \pm 4.11$; $\mathrm{F}[1,490]=7.8, P=0.006)$. Scores of $\geq 5$ on the PSQI indicated bad sleepers (20). Only $4 \%$ of the sample scored $<5$ at baseline, and 5.3\% scored $<5$ at the 1-year assessment, suggesting significant presence of poor sleep quality in this sample at both assessments. The PSQI has 7 component scores that measure various aspects of sleep. Table 1 shows detailed information on the sleep quality of participants at baseline and 1 year. In general, for each of the component scores, participants reported more improvement, i.e., scored lower, at 1 year than at baseline, with statistically significantly less severe sleep problems at 1 year on all domains except for daytime dysfunction and use of sleep medication. Although daytime dysfunction did not change, use of sleep medication increased significantly from the baseline assessment to the 1-year assessment. 
Pain-The Pain Rating Index scale of the McGill Pain Questionnaire showed a small but significant reduction in pain at 1 year compared with baseline (baseline mean \pm SD $1.68 \pm$ $0.68,1$-year mean $\pm \mathrm{SD} 1.45 \pm 0.68 ; \mathrm{F}[2,460]=20.67, P<0.001$ ). Given the range from 0 6 with 6 indicating severe pain, participants reported relatively low levels of pain throughout the study. In previous research of patients with FMS, mean \pm SD PSQI scores ranged from $2.1 \pm 0.3$ to $2.3 \pm 0.08(21,22)$.

Depression scores-At baseline, participants scored in the depressed range, according to the CES-D cutoff score of 19 suggested for populations with FMS pain (mean \pm SD $19.79 \pm$ 11.53) (23), with $49.7 \%$ of the sample at or above this cutoff score. At the 1-year followup, mean CES-D scores were below the cutoff (mean \pm SD $14.78 \pm 9.68$ ), with $30.5 \%$ of participants scoring $\geq 19$. The difference was statistically significant $(\mathrm{F}[1,491]=115.4, P<$ $0.001)$.

Physical functioning-At baseline, participants scored a mean \pm SD of $1.64 \pm 0.71$ on a $0-3$-point scale, where higher scores indicated better functioning; at 1 year it was $1.71 \pm$ 0.70 . These scores were not statistically significantly different, suggesting consistency across the 2 time periods in participants' abilities to carry out basic household tasks such as shopping, vacuuming, and yard work.

\section{Correlational analyses}

Table 2 shows the correlations among all variables in the study. For the main study variables (sleep, pain, physical functioning, and depression), baseline and 1-year data are shown. Younger women fared better on all symptoms over time, except for 1-year pain and 1-year depression, which did not correlate with age. Income had small correlations with all variables except for 1-year sleep. Other noteworthy correlations are those between sleep and ethnicity: whites tended to report better sleep than nonwhites, and education correlated with the 3 main variables, with higher education associated with better outcome overall.

\section{Longitudinal prediction analyses}

Building on the work of Nicassio and Wallston (10), we conducted a path analysis to examine hypothesized causal relationships among pain, sleep, physical functioning, and depression at baseline and 1 year. The analysis was conducted using LISREL 8.8 (24), which tests theoretical causal models based on the observed pattern of relationships (e.g., correlations) among a set of measured variables. This type of path analysis offers several advantages over traditional regression equation analysis, including more accurate regression estimates, reduced likelihood of spurious findings, and the ability to handle missing data.

Moreover, the hypothesized causal model can be evaluated based on how closely it matches the observed pattern of relationships among measured variables. Two goodness-of-fit statistics were used to evaluate the causal model in this study: the chi-square statistic and the root mean square error of approximation (RMSEA) (25). The chi-square statistic measures the absolute fit between the hypothesized model and the observed pattern of relationships among measured variables. A nonsignificant chi-square statistic suggests there is no difference between the hypothesized and observed patterns of relationships and therefore, the hypothesized model is acceptable. The RMSEA statistic adjusts the measure of absolute fit based on the complexity of the hypothesized model, with more complex models receiving a penalty. Smaller values of RMSEA indicate better model fit, with values $<0.06$ representing acceptable model fit (26).

All baseline measures were modeled as predictors and all of the 1-year measures were modeled as dependent variables. In addition, the following demographic and disease 
variables showed significant zero-order correlations with the dependent variables and were included in the path model as predictors: age, ethnicity $(0=$ white, $1=$ nonwhite), level of education, income, marital status $(0=$ not married, $1=$ married $)$, work status $(0=$ not working, $1=$ working), and length of time with FMS symptoms. The data from all study participants $(n=600)$ were included, and missing values were handled using full information maximum likelihood, which is superior to traditional methods of handling missing data (e.g., listwise or pairwise deletion) (27).

Regression equation paths from all predictors to all dependent variables were estimated. In addition, the correlations among all predictor variables were estimated as well as the correlations among all dependent variables. The resulting path model is shown in Figure 1. For the sake of clarity, only significant regression equation paths $(P<0.05)$ are shown. Age, ethnicity, income, and marital status were included in the model, but did not significantly predict any of the dependent variables; therefore, they are not shown in the figure.

As the figure shows, both baseline sleep quality $(\beta=0.49)$ and education $(\beta=-0.10)$ were significant predictors of 1-year sleep quality, accounting for $35 \%$ of the variance. More education predicted better sleep quality.

Baseline pain ( $\beta=0.31)$ and sleep quality $(\beta=0.13)$ were significant predictors for pain at 1 year, along with the length of time since FMS symptoms began $(\beta=0.09)$. These 3 predictors accounted for $22 \%$ of the variance in pain at 1 year. Greater sleep disturbance at baseline in addition to more time since the onset of FMS symptoms predicted greater pain at 1 year.

Physical functioning at 1 year was predicted by baseline physical functioning $(\beta=0.38)$ and pain $(\beta=-0.13)$, accounting for $27 \%$ of the variance. Greater pain at baseline predicted less physical functioning at 1 year. Finally, depression at 1 year was predicted by baseline depression $(\beta=0.45)$, baseline physical functioning $(\beta=-0.10)$, and work status $(\beta=$ -0.08 ), accounting for $31 \%$ of the variance in 1-year depression. Greater baseline physical functioning and being employed predicted less depression at 1 year.

The hypothesized model showed close fit to the data $\left(\chi^{2}[11, \mathrm{n}=600]=13.42, P=0.27\right.$, RMSEA $=0.019$ ). This model suggests a specific causal sequence involving sleep, pain, physical functioning, and depression among patients with FMS. Specifically, greater sleep disturbance predicts greater pain, which then predicts poorer physical functioning. Poorer physical functioning in turn predicts greater depression.

Because the item on the PSQI that measures use of sleep medication showed an increase from the baseline assessment to the 1-year assessment (see Table 2), we ran longitudinal prediction analyses with this 1-item variable removed from the measure and included it as a covariate. There were no changes to the model as a whole, with minimal and not significant changes to the coefficients.

\section{DISCUSSION}

As hypothesized, our findings agree with those of Affleck et al (11), in that sleep precedes pain in fibromyalgia, even with different methodologies and time measures. Whereas Affleck et al (11), using ecological momentary assessment over a 1-week period, found that sleep predicted pain in patients with FMS, the present study, using traditional self-report measures of pain at 2 points during a 1-year period, reached the same conclusion.

This longitudinal study suggests a causal sequence of symptom impact. Specifically, in the present model no baseline variable predicted 1-year sleep, baseline sleep predicted 1-year 
pain, baseline pain predicted 1-year physical functioning, and baseline physical functioning predicted 1-year depression. Clearly, sleep problems among patients with FMS impact symptoms in the long term and deserve more attention both in research and in clinical practice, because they seem to initiate a cascade of symptoms that lead to depression, a serious and prevalent problem in this population. The importance of sleep in the symptomatology of FMS has been identified by sleep researchers as well (2). However, the present study measured these variables over 2 time periods, measuring baseline predictors of 1 -year outcomes instead of various and continuous time periods. Therefore, it is not clear whether these findings truly reflect what would be seen over time if these participants were followed for longer periods and measured repeatedly.

Only 1 previous study with a small sample size $(n=16)$ has reported detailed information on the sleep quality of patients with FMS (28). Our larger sample provides greater support for generalizing findings regarding the characteristics and effects of sleep quality among patients with FMS. Although sleep quality was significantly better at the 1-year assessment, it remained poor, indicating poor sleep may be a chronic problem, with dysfunction during the day showing no improvement. This is further supported by literature indicating that, despite receiving 6-8 hours of sleep, patients with FMS wake up stiff, fatigued, and in pain (29). This suggests that improving sleep quality in this population would decrease pain, possibly also impacting functioning and depression, and that the focus should be on quality of sleep, not quantity.

For depression, pain, and sleep, patients improved by the 1-year assessment. We considered whether these improvements were related to the intervention. The intervention consisted of a social support group, a social support and education about FMS group, and a no treatment control group. However, the intervention groups did not differ from the control group in any of these variables, i.e., the changes in symptoms occurred in the presence of, but not because of, the intervention. Although these findings could represent a simple regression to the mean and should be examined in further research, our findings are consistent with those of other studies indicating that, although FMS symptoms may decrease in severity (30), patients still score within clinical ranges across time $(1,31)$.

These conclusions do not hold as strongly for depression in this sample. The improvements in depression at followup were noteworthy, both as overall means and as proportions that scored above and below the clinical cutoff. Although almost 50\% of participants scored at the depressed range at baseline, that percentage dropped to $30.5 \%$ at 1 year. Although the rates remained high, this drop is both statistically and clinically significant. It could be that there was a placebo effect, because all participants were in a research study. Also noteworthy was the role of depression in the path model; specifically, baseline depression did not contribute to pain, physical functioning, or sleep at 1 year. Our findings suggest that depression may be the end result of a process that begins with sleep problems.

Interestingly, physical functioning did not change from baseline to the 1-year assessment as the other variables did. Scores were consistently average ( 1.7 out of a 4-point scale) at baseline and 1 year. Furthermore, only baseline pain predicted physical functioning at the 1year assessment, with a very small effect size. Physical functioning did predict depression in this sample. This finding was not surprising, although it was not hypothesized. Research has suggested for some time that physical disability predicts later depression (32). The model of depression by Lewinsohn et al (33) suggests that circumstances that disrupt adaptive functioning increase vulnerability to depression. For instance, the individual may be more susceptible to depression as a result of not being able to engage in valued activities such as self-care. 
When interpreting these results, it is important to keep in mind that our sample was highly educated and mostly white. Therefore, generalizing these findings to other populations entails some risks. Also, this sample reported elevated levels of sleep disturbance. Therefore, it would be difficult to determine whether similar results would be elicited in a sample with varying levels of sleep quality. The present study used self-report measures to assess depression, pain, and sleep. These measures may not reflect the symptoms as accurately as objective measures, although subjective reports of sleep quality have been corroborated with objective data for this population (34). Future studies would benefit from including objective measures of these variables. Some studies have used electronic sleep diaries $(9,11)$ or actigraphy $(2,34)$ to study FMS pain and sleep. However, this methodology becomes more difficult when studying larger samples over a longer period of time. Also, all measures assessed symptoms over the past week except for the sleep measure, which assessed symptoms over the past month. This difference in measurement may result in discrepancies, because symptoms may have been very different over the past month and in the past week. However, the correlations between sleep and other variables were similar to those among other indices of symptoms. Finally, all findings should be interpreted with caution, given the long time frame between both assessments. It is possible that there were fluctuations in each variable that were not captured by the 2 assessment periods, instead of a gradual increase or decline suggested by the data. Future studies should attempt more frequent assessments to gain a better understanding of the course of these problems.

Alternately, a strength of the present study was the large sample that was followed over time. Our design allowed us to include more variables than smaller studies because the latter have lower power. Therefore, the present study was able to determine the impact of the main study variables in the presence of relevant demographic and disease variables. In conclusion, the present study highlights the importance of further research in sleep among this patient population, given the findings of the path model.

\section{Acknowledgments}

Supported by the NIH (grants AR-40423, AR-08409, and AR-44020).

\section{REFERENCES}

1. Harding S. Sleep in fibromyalgia patients: subjective and objective findings. Am J Med Sci. 1998; 315:367-376. [PubMed: 9638893]

2. Shaver JL, Lentz M, Landis CA, Heitkemper MM, Buchwald DS, Woods NF. Sleep, psychological distress, and stress arousal in women with fibromyalgia. Res Nurs Health. 1997; 20:247-257. [PubMed: 9179178]

3. Anch AM, Lue FA, MacLean AW, Moldofsky H. Sleep physiology and psychological aspects of the fibrositis (fibromyalgia) syndrome. Can J Psychol. 1991; 45:179-184. [PubMed: 1873756]

4. Korszun A, Young EA, Engleberg NC, Brucksch CB, Greden JF, Crofford LA. Use of actigraphy for monitoring sleep and activity levels in patients with fibromyalgia and depression. J Psychosom Res. 2002; 52:439-443. [PubMed: 12069867]

5. Melzack R, Loeser JD. Phantom body pain in paraplegics: evidence for a central "pattern generating mechanism” for pain. Pain. 1978; 4:195-210. [PubMed: 273200]

6. Bryant PA, Trinder J, Curtis N. Sick and tired: does sleep have a vital role in the immune system? Nat Rev Immunol. 2004; 4:457-467. [PubMed: 15173834]

7. Kato M, Phillips BG, Sigurdsson G, Narkiewicz K, Pesek CA, Somers VK. Effects of sleep deprivation on neural circulatory control. Hypertension. 2000; 35:1173-1175. [PubMed: 10818083]

8. Johnson LC, Slye ES, Dement W. Electroencephalographic and autonomic activity during and after prolonged sleep deprivation. Psychosom Med. 1965; 27:415-423. [PubMed: 5837316] 
9. Hamilton NA, Catley D, Karlson C. Sleep and the affective response to stress and pain. Health Psychol. 2007; 26:288-295. [PubMed: 17500615]

10. Nicassio PM, Wallston KA. Longitudinal relationships among pain, sleep problems, and depression in rheumatoid arthritis. J Abnorm Psychol. 1992; 101:514-520. [PubMed: 1500608]

11. Affleck G, Urrows S, Tennen H, Higgins P, Abeles M. Sequential daily relations of sleep, pain intensity, and attention to pain among women with fibromyalgia. Pain. 1996; 68:363-368. [PubMed: 9121825]

12. Moldofsky H, Scarisbrick P. Induction of neurasthenic musculoskeletal pain syndrome by selective sleep stage deprivation. Psychosom Med. 1975; 38:35-44. [PubMed: 176677]

13. Buysse DJ, Reynolds CF III, Monk TH, Berman SR, Kupfer DJ. The Pittsburgh Sleep Quality Index: a new instrument for psychiatric practice and research. Psychiatry Res. 1989; 28:193-213. [PubMed: 2748771]

14. Melzack R. The McGill Pain Questionnaire: major properties and scoring methods. Pain. 1975; 1:277-299. [PubMed: 1235985]

15. Byrne M, Troy A, Bradley LA, Marchisello PJ, Geisinger KF, Van der Heide LH, et al. Crossvalidation of the factor structure of the McGill Pain Questionnaire. Pain. 1982; 13:193-201. [PubMed: 6214754]

16. Radloff LS. The CES-D Scale: a self-report depression scale for research in the general population. Appl Psychol Meas. 1977; 1:385-401.

17. Burckhardt CS, Clark S, Bennett RM. The Fibromyalgia Impact Questionnaire: development and validation. J Rheumatol. 1991; 18:728-733. [PubMed: 1865419]

18. Oliver K, Cronan TA, Walen HR, Tomita M. Effects of social support and education on health care costs for patients with fibromyalgia. J Rheumatol. 2001; 28:2711-2719. [PubMed: 11764222]

19. Wolfe F, Smythe HA, Yunus MB, Bennett RM, Bombardier C, Goldenberg DL, et al. The American College of Rheumatology 1990 criteria for the classification of fibromyalgia: report of the Multicenter Criteria Committee. Arthritis Rheum. 1990; 33:160-172. [PubMed: 2306288]

20. Carpenter JS, Andrykowski MA. Psychometric evaluation of the Pittsburgh Sleep Quality Index. J Psychosom Res. 1998; 45:5-13. [PubMed: 9720850]

21. Lautenbacher S, Rollman GB, McCain GA. Multi-method assessment of experimental and clinical pain in patients with fibromyalgia. Pain. 1994; 59:45-53. [PubMed: 7854801]

22. Perry F, Heller PH, Levine JD. Differing correlations between pain measures in syndromes with or without explicable organic pathology. Pain. 1988; 34:185-189. [PubMed: 3174156]

23. Turk DC, Okifuji A. Detecting depression in chronic pain patients: adequacy of self-reports. Behav Res Ther. 1994; 32:9-16. [PubMed: 8135727]

24. Joreskog, KG.; Sorbom, D. LISREL, version 8.8 [computer software]. Chicago: Scientific Software International; 2008.

25. Steiger JA. Structural model evaluation and modification: an interval estimation approach. Multivariate Behav Res. 1990; 25:173-180.

26. Hu LT, Bentler PM. Cutoff criteria for fit indexes in covariance structure analysis: conventional criteria versus new alternatives. Struct Equation Model. 1999; 6:1-55.

27. Enders CK. The performance of the full information maximum likelihood estimator in multiple regression models with missing data. Educ Psychol Meas. 2001; 61:713-740.

28. Agargun MY, Tekeoglu I, Gunes A, Adak B, Kara H, Ercan M. Sleep quality and pain threshold in patients with fibromyalgia. Compr Psychiatry. 1999; 40:226-228. [PubMed: 10360619]

29. Moldofsky H. Sleep and fibrositis syndrome. Rheum Dis Clin North Am. 1989; 15:91-103. [PubMed: 2644681]

30. Kennedy M, Felson DT. A prospective long-term study of fibromyalgia syndrome. Arthritis Rheum. 1996; 39:682-685. [PubMed: 8630121]

31. Wolfe F, Anderson J, Harkness D, Bennet RM, Caro XJ, Goldenberg DL, et al. Health status and disease severity in fibromyalgia: results of a six-center longitudinal study. Arthritis Rheum. 1997; 40:1571-1579. [PubMed: 9324010] 
32. Kaplan GA, Roberts RE, Camacho TC, Coyne JC. Psychosocial predictors of depression: prospective evidence from the human laboratory studies. Am J Epidemiol. 1987; 125:206-220. [PubMed: 3812429]

33. Lewinsohn, PM.; Hoberman, HM.; Teri, L.; Hautzinger, M. An integrated theory of depression. In: Reiss, S.; Bootzin, RR., editors. Theoretical issues in behavior therapy. New York: Academic Press; 1985. p. 331-359.

34. Landis CA, Frey CA, Lentz MJ, Rothermel J, Buchwald D, Shaver JL. Self-reported sleep quality and fatigue correlates with actigraphy in midlife women with fibromyalgia. Nurs Res. 2003; 52:140-147. [PubMed: 12792254] 


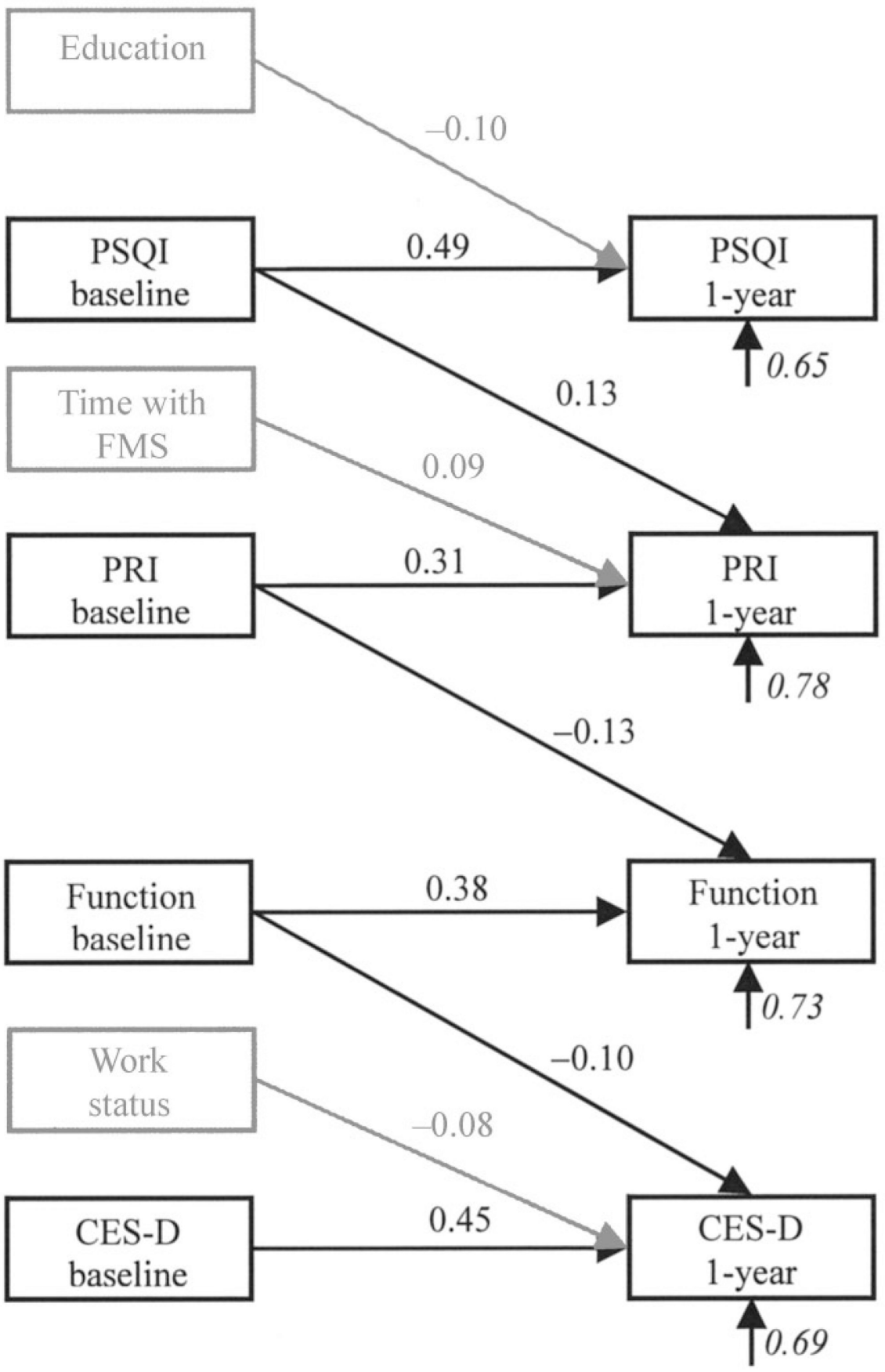

Figure 1.

Path model of sleep, pain, physical functioning, and depression. Italicized coefficients are error terms, representing the proportion of variation in the variable not accounted for by the model; $P<0.05$ for all coefficients. PSQI $=$ Pittsburgh Sleep Quality Index; FMS = fibromyalgia syndrome; PRI = Pain Rating Index; Function = physical functioning; CES-D $=$ Center for Epidemiologic Studies Depression Scale. 
Table 1

Pittsburgh Sleep Quality Index sleep component scores at baseline and 1 year

\begin{tabular}{|lcccc|}
\hline Component & $\begin{array}{c}\text { Baseline, } \\
\text { mean } \pm \text { SD }\end{array}$ & $\begin{array}{c}\text { 1 year, } \\
\text { mean } \pm \text { SD }\end{array}$ & F statistic & $P$ \\
\hline Sleep quality, 0 = very good to $3=$ very bad & $1.66 \pm 0.80$ & $1.52 \pm 0.79$ & $\mathrm{~F}(1,488)=13.39$ & $<0.001$ \\
Sleep latency (time to fall asleep), $0=<15$ minutes to $3=>60$ minutes & $1.55 \pm 1.05$ & $1.44 \pm 1.12$ & $\mathrm{~F}(1,490)=6.58$ & 0.011 \\
Sleep duration (hours of sleep), $0=>7$ hours to $3=<5$ hours & $1.34 \pm 0.93$ & $1.21 \pm 0.90$ & $\mathrm{~F}(1,490)=11.00$ & 0.001 \\
Sleep efficiency (percentage of time asleep while in bed), 0 = >84\% to 3=<65\% & $1.41 \pm 1.23$ & $1.09 \pm 1.26$ & $\mathrm{~F}(1,490)=25.81$ & $<0.001$ \\
Sleep disturbances, $0=$ not during the past month to 3 = 3 times/week & $1.89 \pm 0.63$ & $1.73 \pm 0.66$ & $\mathrm{~F}(1,490)=20.87$ & $<0.001$ \\
Use of sleep medication, $0=$ not during the past month to 3 = 3 times/week & $1.78 \pm 1.37$ & $1.97 \pm 1.13$ & $\mathrm{~F}(1,488)=9.04$ & 0.003 \\
Daytime dysfunction, $0=$ not during the past month to 3 = 3 times/week & $1.61 \pm 0.79$ & $1.55 \pm 0.82$ & $\mathrm{~F}(1,489)=2.49$ & 0.115 \\
\hline
\end{tabular}




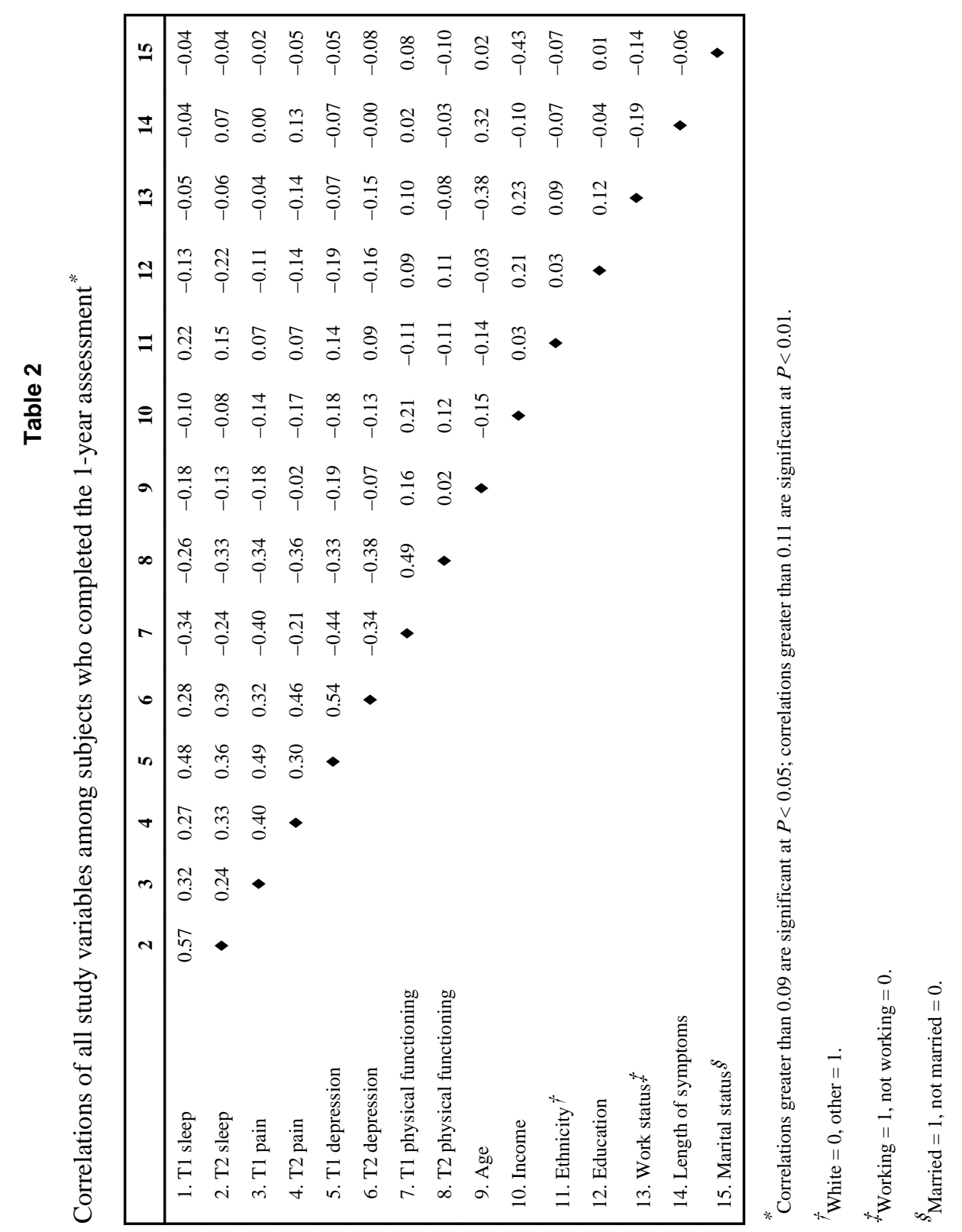

Arthritis Rheum. Author manuscript; available in PMC 2013 June 25. 